\title{
How does evidence help identify new forms of support for older people?
}

Forms of support available to older people in different societies are changing as new means of support are developed and introduced, whose effectiveness may be assumed, but where their characteristics and uses in context may need to be examined and evaluated.

The introduction and implementation of pension policies to more societies may be assumed to support higher levels of wellbeing and indeed happiness among the older population. Park's research aims to examine the relationship between pensions policy and its impact on the happiness of older people. The data of people aged 65 and over from the Korean Longitudinal Study of Ageing actually show a negative relationship between receiving the basic old age pension and the happiness of older people. Analysis suggests that introducing such a social policy needs to take account of contextual factors as well as its simple presence or absence, to include perceptions of its sufficiency and processes involved in accessing it such as means-testing. It suggests that changing the premises of this system to build in progressive values can align the effects of such policies more closely to the needs and circumstances of older recipients.

Another major contextual change affecting forms of support is the internet, widely expected in its early introduction to benefit many isolated groups including older people and to mitigate social, health and wellbeing. A considerable and recent literature investigated by Beneito-Montegut et al. has investigated the role and effects of the internet on social isolation in later life. Their review of empirical literature published since 2000 finds relatively little well-founded evidence to demonstrate what its effects may be on social isolation and loneliness. They suggest that the evidence base here needs to be strengthened by ensuring study designs include robust methodological and theoretical frameworks, more longitudinal research and that they offer more precise and nuanced descriptive measures both of social isolation and loneliness and of ways in which the internet mediates social interaction.

Another technological service innovation assumed to strengthen care options is telecare, where novel features demand new related roles, including in the non-statutory sector. Naick's reports a first study to examine one such new role in England, which is of care navigators and telecare installers increasingly used to facilitate telecare provision for older people in terms of care navigators' experiences from recruitment and training to service outcomes, when assessing older adults for telecare. Naick's study uses semi-structured interviews to examine the experience of a purposeful sample of 11 participants from five non-statutory organisations. Again, here contextual and organisational factors are seen to impact on how their practice develops, highlighting support for autonomy, exchanging knowledge, enabling practice to evolving and sustaining performance. The findings suggested that the strategic placement of care navigators could help match telecare assessment better to locality demand when facilitating hospital discharges. While home assessment may be assumed to be a gold standard for care navigator practice, this study suggests a more sustainable model for organising care navigators' work within hospital teams and in providing home assessments.

Traditional forms of social support for older people including those of long-term church members may also be changing and be perceived differently. Sta. Maria et al. have studied the quality and degree of supportiveness of relationships in the social networks of older Filipino church members. Their study used a qualitative semi-structured interview design with a purposive sample of Filipino older people. Their analysis drew on social convoy theory to track how types of 
support confirmed ways in which social roles of older people were seen to be changing and that drawing on support from within these social networks may have helped them sustain their meaningful engagement in activities.

Finally, an international trend is for an increasing range of specialist healthcare support to be required by older people living in care homes, with and without nursing as their needs become more complex, but there is not yet a consensus on how these may be appropriately and efficiently delivered. Pressures on the UK National Health Service are growing as this population grows and encounters multiple health and support needs, stemming from progressive chronic conditions which may also bring sensory deficits, depression and dementia. However, the service provided to older people who live in care homes may often be relatively poor, perhaps reflecting the deficits of clinical leadership and challenges for translating evidence based care to practice. The review carried out by these authors overviewed the range of services to evidence their design, scope and effectiveness to indicate what types of information to specify in service reports. Their findings underlined the need for clear specification of and close adherence of care staff to healthcare interventions and, as found in some of the other studies reported in this journal issue, suggested the need to understand effectiveness in the implementation context. This would influence access to regulatory procedures, additional training, effective communication of aims and care home staff "buy-in" to any interventions. The authors argue for more robust evidence to be provided by developing models based on meta-regression and comparing mixes of components to help explain reasons for some approaches' lack of effectiveness. They suggest the considerable locality variation in healthcare provision to care homes means there is not yet any comparator of "standard care" for trials studies to inform evaluations of different healthcare provision models to care homes but suggest a potential economic model of relative cost-effectiveness, to support more rigorous trials of more complex healthcare provision in care homes.

We are still building an evidence base about forms of support for older people, which is now having to simultaneously take into account local and international variations in practices and values, in older people's demography, in relationships between social groups and between people and novel relational and assistive technologies. Addressing this challenge is seen here to demand correspondingly new approaches to and standards if we are to build an evidence base that can be fit for purpose.

\section{About the editor}

Professor Fiona Poland is the Editor of Quality in Ageing and Older Adults from the University of East Anglia, January 2018. 